\title{
A Rate Adaptation Algorithm for IEEE 802.11 WLANs Based on MAC-Layer Loss Differentiation
}

\author{
Qixiang Pang, Victor C.M. Leung \\ Department of Electrical and Computer Engineering \\ The University of British Columbia \\ Vancouver, BC, Canada V6T 1Z4
}

\author{
Soung C. Liew \\ Department of Information Engineering \\ The Chinese University of Hong Kong \\ Shatin, Hong Kong
}

\begin{abstract}
In a WLAN subject to variable wireless channel conditions, rate adaptation plays an important role to more efficiently utilize the physical link. However, the existing rate adaptation algorithms for IEEE 802.11 WLANs do not take into account the loss of frames due to collisions. In a WLAN with coexistence of multiple stations, two types of frame losses due to (a) link errors and (b) collisions over the wireless link can coexist and severely degrade the performance of the existing rate adaptation algorithms. In this paper, we propose a new automatic rate fallback algorithm that can differentiate the two types of losses and sharpen the accuracy of the rate adaptation process. Numerical results show that the new algorithm can substantially improve the performance of IEEE 802.11 WLANs.
\end{abstract}

Keywords - IEEE 802.11, WLAN, DCF, loss differentiation, rate adaptation, automatic rate fallback

\section{INTRODUCTION}

The use of wireless local area networks (WLANs) in homes, offices, and public areas has been spreading quickly. The dominating WLAN standards have been defined by the IEEE 802.11 working group, with their success propelled by the WiFi Alliance [1][2].

Most IEEE 802.11 WLAN devices use the Distributed Coordination Function (DCF) specified in the standard [2] to coordinate channel access by means of carrier-sense multiple access with collision avoidance (CSMA/CA). In DCF, when multiple frames are transmitted simultaneously by different stations, a collision occurs, which destroys all the transmitted frames. To resolve collisions, the stations employ a binary slotted exponential backoff algorithm and retransmission scheme. Two access procedures are defined in $802.11 \mathrm{DCF}$, the default basic access and the optional RTS/CTS access.

Current WLAN products support more than one modulation types and data rates. For example, in $802.11 \mathrm{~b}, 4$ data rates, 1 Mbps, $2 \mathrm{Mbps}$, 5.5 Mbps, and $11 \mathrm{Mbps,}$ are supported [3]. However, a higher data rate does not necessarily yield a higher throughput. Only when the channel condition is good, does a higher data rate give a higher throughput. In a realistic WLAN environment, the channel condition can vary dynamically due to multi-path interference, and movements of stations, etc. To accommodate different channel conditions, rate adaptation (or auto rate) is commonly employed. This is realized by rate adaptation algorithms that dynamically adjust the modulation mode and data rate to optimize performance when channel condition changes.
Automatic Rate Fallback (ARF) is the original rate adaptation algorithm for WLAN implemented by Lucent in its WaveLAN-II product [4]. ARF is also the most successful rate adaptation algorithm so far. ARF and other algorithms with a similar concept have been widely implemented in many WLAN products although it is not included in the IEEE standard [2]. In ARF, the sender deduces the channel condition by measuring the numbers of consecutively successful and failed transmissions. The sender adjusts its modulation mode and data rate in accordance with these measurements.

However, the design of ARF has not taken into account possible frame losses due to collisions. It assumes that all frame losses are due to channel errors. ARF lowers the data transmission rate whenever consecutive frame losses occur. It is appropriate when there is only one station. When a WLAN has a number of active stations, however, frequent collisions may happen and ARF may lose its effectiveness. As a matter of fact, if losses are due to collisions, reducing the data transmission rate will certainly reduce throughput. In such cases, not only the transmission time of a data frame, but also the collision period, are longer. Worse, if a station using a higher data rate collides with another one using a lower data rate, both stations will experience the same collision period determined by the lower data rate. Other existing rate adaptation algorithms [5]-[10] have the same disability.

The reason for this disability might come from the fact that traditionally rate adaptation was used in telecommunication networks where traffic transmissions are based on multiple access technique without contention (e.g., TDMA, FDMA and CDMA). In contrast, channel access in 802.11 WLANs is fully distributed and contention based. When transplanting the rate adaptation algorithms from other communication systems, the characteristics of the WLAN access procedure should have been properly taken into account.

In this paper, we propose a new auto rate algorithm called loss-differentiating-ARF (LD-ARF) that is suitable for a realistic WLAN environment where both collision losses and link error losses can coexist, and demonstrate its effectiveness through extensive performance evaluations.

The organization of the remainder of this paper is as follows. Section 2 reviews the problems in the existing autorate algorithms. The disability of the popular ARF algorithm when there are multiple stations is discussed. In Section 3, a new loss differentiating ARF algorithm is proposed. The new 
algorithm involves MAC layer changes only. The MAC layer protocol is modified to have loss differentiation ability. In Section 4, we design various simulation scenarios to evaluate the performance of LD-ARF. Simulation results are presented to show that the new LD-ARF algorithm improves throughput significantly over ARF when there are multiple active stations. Section 5 concludes this paper and discusses possible future work. The appendix gives the physical layer properties of 802.11b WLANs used in our performance evaluations.

\section{Problems with The Existing Auto-Rate ALGORITHMS}

\section{A. Physical Features of IEEE 802.11 WLANs}

In this paper, we use $802.11 \mathrm{~b}$ [3] as an example to evaluate the performance of the auto-rate algorithms because $802.11 \mathrm{~b}$ is the most successful and widely used WLAN technology so far and its physical property has been well established in $[11][12][13]$.

$802.11 \mathrm{~b}$ provides 4 physical modes/rates as listed in TABLE I. The bit error rate (BER) vs. signal-to-noise ratio (SNR) relations of the 4 modes have been discussed in [11][12][13] and given in the Appendix.

Figure 1 shows the throughput achieved by a single station transmitting at different data rates under different channel conditions (SNR values). The basic access procedure of 802.11 WLAN was simulated using NS-2 [14]. In the simulations, the station is saturated with UDP type traffic, i.e., it always has data frames to send. The MAC layer payload size is 1000 bytes. The values of other parameters are listed in TABLE II. The measured performance metrics is the MAC layer payload throughput.

TABLE I. $\quad$ 802.11B DATA RATE SPECIFICATIONS

\begin{tabular}{|c|c|c|c|c|}
\hline Data Rate & Code Length & Modulation & $\begin{array}{c}\text { Symbol } \\
\text { Rate }\end{array}$ & $\begin{array}{c}\text { Bits } \\
\text { /Symbol }\end{array}$ \\
\hline $1 \mathrm{Mbps}$ & 11 (Barker Sequence) & BPSK & $1 \mathrm{MSps}$ & 1 \\
\hline $2 \mathrm{Mbps}$ & 11 (Barker Sequence) & QPSK & $1 \mathrm{MSps}$ & 2 \\
\hline $5.5 \mathrm{Mbps}$ & $8(\mathrm{CCK})$ & QPSK & $1.375 \mathrm{MSps}$ & 4 \\
\hline $11 \mathrm{Mbps}$ & $8(\mathrm{CCK})$ & QPSK & $1.375 \mathrm{MSps}$ & 8 \\
\hline
\end{tabular}

TABLE II. NOTATIONS AND PARAMETER VALUES OF 802.11B

\begin{tabular}{|c|l|c|}
\hline Notations & \multicolumn{1}{|c|}{ Descriptions } & Values \\
\hline$C W m i n$ & Min contention window & 32 \\
\hline$C W m a x$ & Max contention window & 1024 \\
\hline MAC & MAC header and checksum & $224 \mathrm{bits}$ \\
\hline DIFS & the time of DIFS & $50 \mu \mathrm{s}$ \\
\hline SIFS & the time of SIFS & $10 \mu \mathrm{s}$ \\
\hline$A C K$ & ACK frame size & $112 \mathrm{bits}$ \\
\hline$R T S$ & RTS frame size & $160 \mathrm{bits}$ \\
\hline$C T S$ & CTS frame size & $112 \mathrm{bits}$ \\
\hline PHY & overhead in physical layer & $192 \mu \mathrm{s}$ \\
\hline DataRate & Physical rate for data frame & See TABLE I \\
\hline BasicRate & Physical rate for control frame & Same as DataRate \\
\hline
\end{tabular}

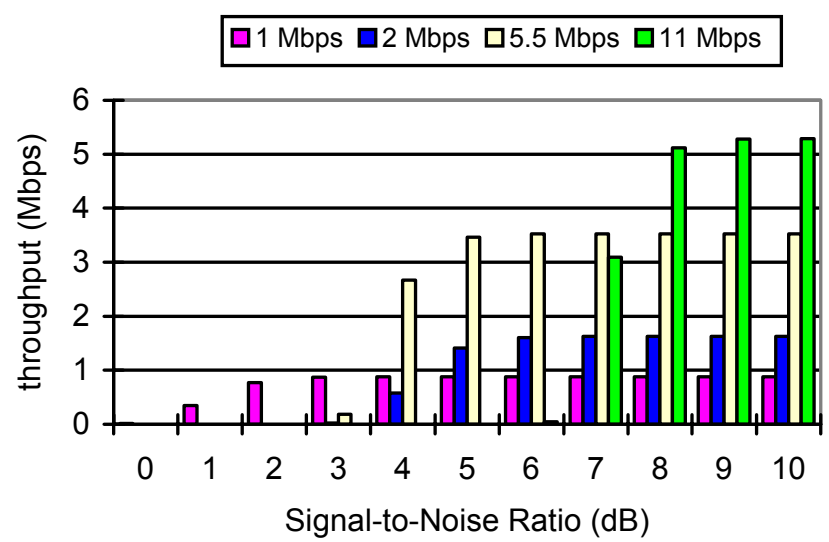

Figure 1. $802.11 \mathrm{~b}$ throughput versus SNR and data rates

It can be seen from Figure 1 that a higher data rate does not necessarily produce a higher throughput. This is because for a given SNR value, BER generally increases with the increase of data rate. When the SNR value is low, the stations must work at a low data rate. The objective of auto rate algorithms is therefore to dynamically adjust the data rate to achieve optimized performance under variable channel condition. However, when there are multiple stations in one WLAN, the existing auto rate algorithms lose their effectiveness. Assuming all losses are due to channel errors and reducing the data rate when collisions occur may lead to unacceptable performance.

\section{B. Related Work and Existing Rate Adaptation Algorithms}

Among the existing rate adaptation algorithms for 802.11 WLANs, the earliest and the most widely used one is ARF as implemented in Lucent's WaveLAN-II product [4] and described below.

In the ARF algorithm, a discrete set of data rates are used. If the ACK frames for $N_{\text {down }}$ (equals to 1 if the rate has just been increased, otherwise equals to 2, as specified in [4]) consecutive data packets are not received by the sender, then the sender drops the transmission rate to the next lower data rate and a timer (we call it the rate-up timer here) is started. If $N_{u p}$ (equals to 10 as given in [4]) consecutive ACK frames are received or the timer expires, then the transmission rate is raised to the next higher data rate and the timer is stopped. When the rate is increased, the first transmission (also called probing frame) after the rate increase must be successful or else the rate is immediately decreased and the timer is started again.

Many commercial WLAN products have implemented ARF or similar algorithms based on the same concept. This is also probably the only class of auto rate algorithms implemented in practical WLAN products.

Despite the wide application of ARF, it can be shown that ARF can be dysfunctional when multiple stations coexist. We consider a WLAN basic service set (BSS) in which there are a number of stations that can hear each other. The behaviors of the saturated stations in such a WLAN have been well analyzed in [15][16][17]. If we let $p$ be the collision probability of a data frame transmission from one station, the analyses in $[15][16][17]$ suggest that $p$ is independent of the data rate and 
increases strictly monotonically and approaches arbitrarily close to 1 as the number of active stations increases. Therefore, when ARF is enabled in all the stations in a WLAN, these stations will misinterpret frequent collisions as link error losses and reduce their data rates unnecessarily. Even when the channel conditions are good, if there are frequent collisions due to heavy traffic generated by many stations, the stations can not use a higher data rate to achieve a higher throughput due to the misinterpretation of collisions as error losses.

The collisions between the stations bring uncertainty to ARF performance and difficulty to ARF design. By simply adjusting ARF control parameters the problem cannot be solved. The reason is that when a collision loss occurs, the data rate should not be decreased. Since the standard MAC protocol does not provide any mechanism to differentiate between the two types of losses, in order to improve the performance of $\mathrm{ARF}$, both the MAC layer protocol and the ARF algorithm need to be modified.

Besides ARF, there are many other rate adaptation algorithms that have been studied in literature [5]-[10]. We review them briefly below in chronicle order according to their publication dates.

In [5], a Frame Error Rate (FER) based approach was proposed for rate adaptation. Two types of operations using FER information are as follows:

Downscaling - If the FER exceeds some threshold, then switch to the next lower rate.

Upscaling - If the FER falls below a second threshold, probe the link at the next higher rate with a few (usually only 1) frames. If all of them get acknowledged, switch to that rate. To prevent the control algorithm from oscillating between two adjacent rates, the upscale action may be prohibited for some time after a downscale decision.

The parameters (e.g., the time windows and the thresholds) mentioned above are critical for the performance of the FERbased algorithm. It is difficult to get reliable FER statistics in a short window under a variable link condition. Hence, many frames are transmitted at a non-optimal rate. On the other hand, the frame errors can also be caused by collisions. Therefore in case of multiple stations causing many collisions, the algorithm may experience the same problem as ARF.

The Receiver-Based Auto Rate (RBAR) scheme [6] performs rate adaptation at the receiver instead of at the sender. RBAR mandates the use of RTS/CTS mechanism. The receiver of an RTS frame calculates the transmission rate to be used by the sender based on the SNR of the received RTS frame and on a set of SNR thresholds calculated with an a priori wireless channel model. The rate to be used to send the data frame is then returned to the sender in the CTS frame. The RTS, CTS and data frames are modified to include information on the size and rate of the data frame transmission to allow all the nodes within the transmission range to correctly update their NAV. Some flaws exist in RBAR. First, the RTS/CTS access procedure is required even if no hidden terminals are present. The extra overhead incurred by RTS/CTS is well known. Secondly, it requires a priori knowledge of the channel model to calculate the SNR thresholds. Thirdly, it requires the receivers to measure SNR, which may be difficult to realize in low cost WLAN devices. Finally, same as other rate adaptation algorithms, it does not consider possible frame collisions.

Similar to RBAR, the method proposed in [7] makes use of an a priori wireless channel model and measured SNR, but performs rate adaptation using a static PHY mode table indexed by the data frame length and channel SNR, which is pre-established offline according to the a priori channel model. Unlike RBAR, RTS/CTS is not used. The sender dynamically monitors the wireless channel condition and estimates the SNR value at the receiver side, which may not be accurate. Except for saving the RTS/CTS overhead, the method proposed in [7] has similar flaws to RBAR.

Since inexpensive receivers do not have the ability to measure SNR, an approach using the received signal strength (RSS) was studied in [8]. It is also a channel model based method, but the channel condition is measured by the RSS. It can solve some but not all of the problems with SNR based methods. The major shortcoming of this method is the same as that in [7], i.e., it does not provide a proper information feedback mechanism and it estimates the receiver's channel condition (RSS level) at the sender side. To do that, fixed power level of the sender must be assumed, which is not realistic in some cases. Moreover the traffic must be bidirectional and frequent enough for the current channel condition to be estimated promptly. Same as others, the performance evaluations did not consider coexistence of multiple stations.

In [9], after a review of existing mechanisms, the authors proposed a hybrid rate control scheme in which the existing techniques are carefully combined. Both statistical information (FER, throughput) and measured SNR (or RSS) information are collected and utilized. By using a more complex control mechanism, it is possible to overcome some of the problems in existing methods as described above. However, not withstanding its complexity, this method still does not account for the coexistence of multiple stations, since it is just a combination of the existing algorithms.

The adaptive ARF (AARF) algorithm [10] was published at almost the same time as [9]. Unlike ARF, AARF continuously change the threshold $\left(N_{u p}\right)$ value at runtime. When the transmission of the probing frame fails, the data rate is switched back immediately and the threshold $\left(N_{u p}\right)$ is doubled. The threshold is reset to its initial value of 10 when the rate is decreased because of 2 consecutive failed transmissions. In this way, the authors claimed that it works better in a high latency system. However, obviously the AARF still cannot solve the collision problem. To make matter even worse than ARF, when a failed transmission is caused by a collision, doubling $N_{u p}$ can further reduce the chance of using an appropriate rate.

In summary, the existing rate adaptation algorithms [5]-[10] share the common drawback that they do not take MAC frame collisions between different stations into account and assume that all frame losses are due to link errors. In their performance evaluations by simulations or theoretical analyses, they considered only one connection between a sender and a receiver. When many stations coexist and send data frames frequently, many collisions can occur. By neglecting these 
collisions, the performance of existing rate adaptation schemes can be severely degraded under heavy WLAN traffic.

The main contributions of this paper are the proposal of the new LD-ARF algorithm, and the performance evaluations to show that it effectively adapts to combinations of collisions and link errors that occur in practice. Our method involves only minimal modifications to the MAC layer.

In the remaining parts of this paper, we mainly base our discussions on ARF because it is the only one implemented in real products and also because of its simplicity in involving MAC layer functions only. Another advantage of ARF is that although ARF may be not optimal for a specific channel model, compared with those channel-model and SNR based approaches, ARF is less sensitive to variation of channel models and can achieve near-optimal performance for a wider range of channel models. The original ARF algorithm will be used as a benchmark to compare with our new LD-ARF algorithm. However, the essential principle in this paper can be extended to other existing rate adaptation algorithms as well.

\section{DESCRIPTION OF LOSS-DIFFERENTIATING ARF ALGORITHM}

In our preliminary work [18], we have described the lossdifferentiating MAC layer protocol. However, neither the performance of the protocol nor its applications has been given. In this section, firstly, the major concept of this protocol is reviewed. Then the LD-ARF algorithm, which combines the loss-differentiating MAC and the existing ARF algorithm, is proposed. The next section will evaluate its performance.

\section{A. Loss Differentiating MAC Protocol}

The loss-differentiating MAC layer protocol [18] is intended as an enhancement of the 802.11 DCF. There are two access procedures in DCF, basic and RTS/CTS. Figure 2 depicts the two access procedures. The modification to the DCF in the new protocol involves both basic and RTS/CTS access procedures.

RTS/CTS is an optional feature in an 802.11 WLAN and it is useful when the data frame size is very large, the number of stations is very large, or there are hidden terminals. It is a 4way handshake procedure as shown in Figure 2.

The loss differentiation method in the RTS/CTS access procedure is straightforward and the 4-way message exchange sequence is not changed: (i) If both the CTS and then the ACK frames are received at the sender, the transmission was successful. (ii) If the CTS frame is received but the ACK frame is not, the transmission has failed, most likely due to a link error. (iii) If the CTS frame is not received, most likely a collision has occurred. Because RTS and CTS are short and usually transmitted at a low rate, the loss differentiation can be quite effective.

Basic access is the default access in 802.11 DCF. It is a two-way handshake procedure (see Figure 2). In most cases, e.g., when there is no hidden terminal, the default basic access is more efficient than RTS/CTS access. The loss differentiation for basic access is not as straightforward as that for RTS/CTS access. In the original basic access procedure, only when the
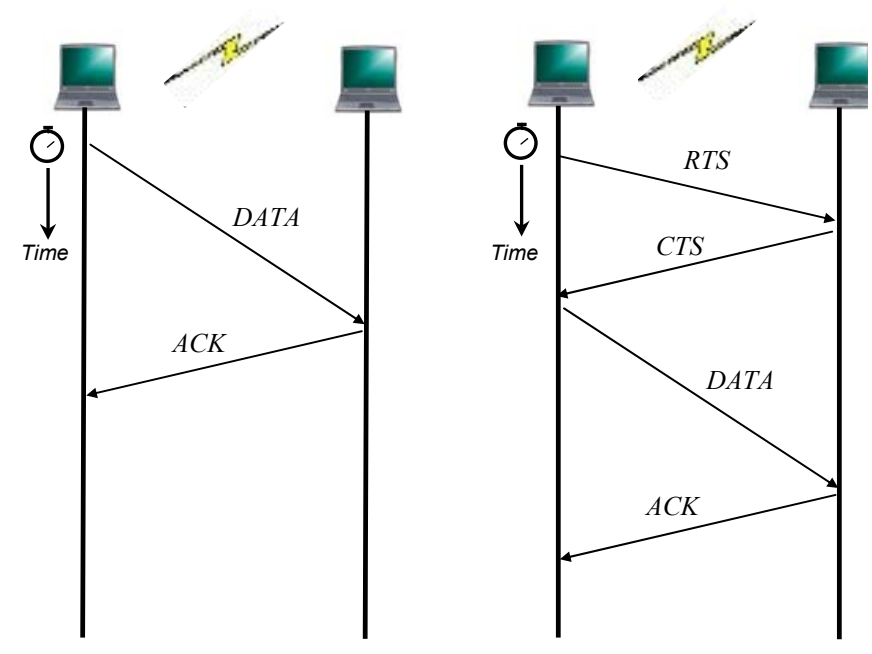

Figure 2. Basic and RTS/CTS access in 802.11 DCF

received data frame is correct, is a feedback message (ACK) sent. When the received data frame is in error, the receiver does not give any response. As explained previously, two reasons, i.e., a collision or link errors, may cause an erroneous frame reception in the basic access procedure, and the sender cannot differentiate between these loss mechanisms and take corrective measure. Therefore, a mechanism must be incorporated so that the receiver can determine the cause of a failed reception and inform the sender accordingly.

The loss differentiation method is based on the following observation. The MAC data frame can be partitioned into two functional parts: header and body. The header contains information such as frame type, source address and destination address. The body contains the payload. If all stations in a WLAN can hear one another (i.e., there is no hidden terminal), a collision occurs only when more than one stations send data frames in the same time slot. In this case, both the header and body will be corrupted. The receiver can neither receive the header nor the payload.

However, if there is only one station sending a data frame and the frame is lost due to link errors, there is a high probability that the receiver will receive the header correctly. This is because in general the header is much shorter than the whole frame. By observing the content in the header, we can obtain information on who has sent the frame and who is the intended receiver.

To verify the correctness of the MAC header, a short checksum field is required for the header of a data frame (Figure 3), in the same manner as the Header Error Control (HEC) field in ATM cell headers [19].

The header checksum field costs extra overhead for the loss differentiation protocol. The total channel occupation time of a data frame includes the transmission times of the data frame and its ACK or NAK frame plus DIFS and SIFS. It can be easily calculated that the overhead due to the extra field to the total time is much less than $1 \%$ in most cases. Therefore the overhead is negligible. 
When a link error occurs, a proper feedback message from the receiver enables the sender to find out what has happened. To notify the sender of the data-frame reception status, a new control frame, $N A K$, is added to the 802.11 DCF. The NAK is used to tell the sender that the data frame transmission has failed and the failure is due to a link error. The structure of NAK frame is the same as that of ACK frame except for a onebit difference in the frame type field of the header. The physical rate to sent NAK frame is also the same as that to ACK frame.

Since the transmission of a NAK occurs only SIFS after the data frame transmission, the sender can know the current link quality very promptly. In this way, it is more efficient than the channel estimation approaches used in the other existing auto rate algorithms reviewed in Section II.

One of the advantages of the new loss-differentiating MAC layer protocol is that the change to the standard DCF function has been minimized. No modification to the PHY layer is needed. Therefore the protocol is easy to implement.

\section{B. Loss Differentiating Automatic Rate Fallback Algorithm}

We have explained that when a collision occurs, the data rate should not be reduced. Therefore the modification to the ARF algorithm is that the data rate is reduced only when a loss of data frame is caused by link errors. Figure 3 describes the new LD-ARF algorithm.

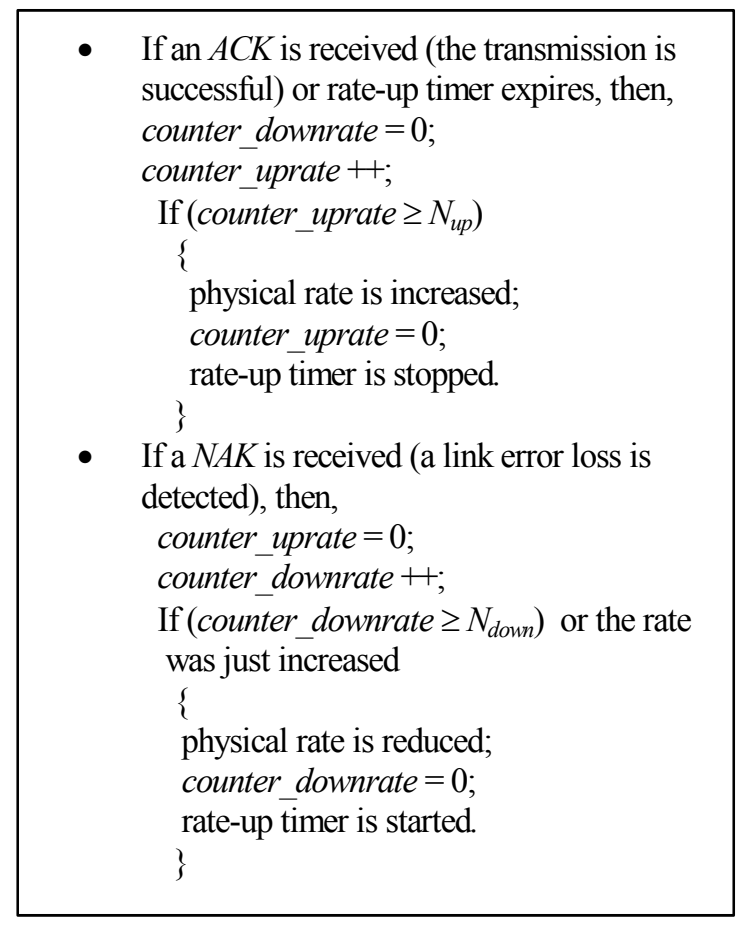

Figure 3. New LD-ARF algorithm

\section{Performance Evaluation of Loss DIFFERENTIATING ARF ALGORITHM}

The Gilbert-Elliot channel model [20][21] has been widely used to model wireless channels subject to burst-errors. Measurements conducted on the wireless channel in an IEEE 802.11 WLAN [22] show that the Gilbert-Elliot model gives a good prediction of WLAN performance. To evaluate the performance of the proposed LD-ARF algorithm under variable conditions and compare with that of the ARF algorithm, in this paper we use an extension model of the original Gilbert-Elliot model. It is a $K$-state Markov channel model as shown in Figure 4. Some properties of the $K$-state Markov model has been studied in [23].

In the $K$-state model, the wireless channel could be in one of the $K$ states from $\mathrm{S}_{0}$ to $\mathrm{S}_{K-1}$. In each state, $\mathrm{S}_{i}$, the corresponding SNR value at each frame transmission is uniformly taken from the range of $\left[\mathrm{x}_{i}, x_{i+1}\right) \mathrm{dB}$, where $x_{i+1}>x_{i}$. The sojourn time in each state follows an exponential distribution (or if discrete time is used, the equivalent Geometric distribution). This model is quite similar to the random walk model, which can be seen as the case where the two communicating WLAN stations approach and depart randomly.

In our simulations, we use a 10 -state channel model. We consider a moderately changing channel model where for each state, $\mathrm{S}_{i,}$, the average time duration is 1 second, and the transition probabilities $t_{i, i-1}=t_{i, i+1}$. For each state, $\mathrm{S}_{i}$, the SNR values (in $\mathrm{dB}$ ) are uniformly distributed between $[i, i+1]$. Therefore the possible SNR values generated by this model are distributed between $0 \mathrm{~dB}$ and $10 \mathrm{~dB}$.

IEEE 802.11b [3] is used in our simulations since it is the most widely used WLAN standard by far. Moreover, its physical property has been well established in [11][12][13]. Furthermore, the WaveLAN-II product in which ARF was originally implemented has similar properties to $802.11 \mathrm{~b}^{1}$. It is more meaningful to use the control parameter values given in WaveLAN-II to examine the performance of the auto rate algorithms. From Figure 1 and Figure 11 (in Appendix), it can be seen that the data rate of $2 \mathrm{Mbps}$ does not generate a better throughput for any SNR value in the range of 0 to 10 . In our simulations for ARF and LD-ARF, we simply by-pass this data rate.

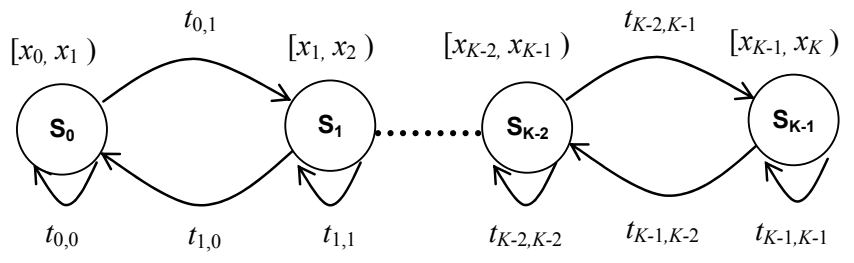

Figure 4. A K-state Markov chain to model the wireless channel variation

${ }^{1}$ WaveLAN-II [4] supports four data rates: 1 Mbps, 2 Mbps, 5 Mbps and 8
Mbps. 


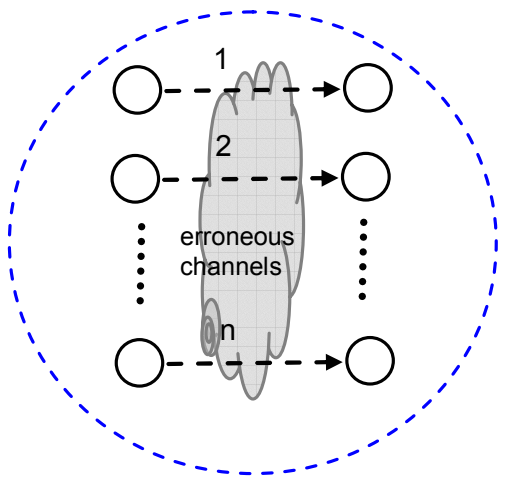

(a) $\quad \rightarrow \quad$ Data traffic

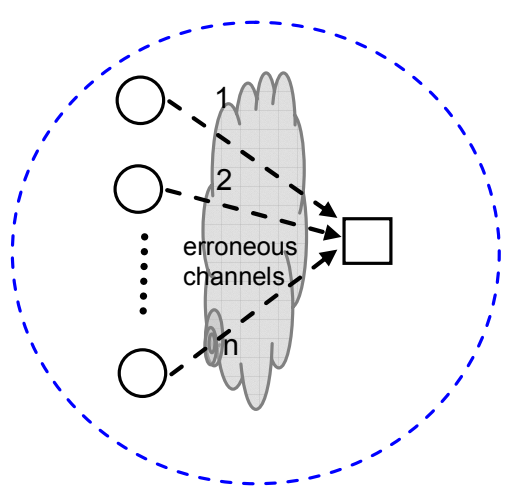

(b)

Figure 5. Two different but performance equivalent WLAN topologies

In the simulation scenarios, all stations are saturated, but we believe the conclusions derived from the simulation results are applicable to non-saturated stations as well. The network topologies are shown in Figure 5. Assume there is no hidden terminal and the stations are close enough that when they transmit frames simultaneously, the resulting collision destroys the frames involved. The data frame payload size is 1000 bytes.

The data frame error rate $(F E R)$, the data frame MAC header error rate $(H E R)$, and the control frame error rates are determined respectively by their sizes, their rates, and the SNR values generated from the 10 -state channel model when the frame is sent. The MAC header and body parts are transmitted at the same data rate. The basic rates for the control frames follow the rules given below. For a RTS frame transmission, its rate is the same as the sending station's data rate for a data frame. For ACK, NAK and CTS, their sending rates are the same as the rate used for the received data frame or RTS frame. Note that if the basic rate or the header part rate is lower than the data frame rate, the loss differentiating MAC protocol can be more effective. Therefore these settings may not yield the best performance for the LD-ARF. We are going to show that even so the LD-ARF can achieve significant performance gain over ARF.

The value of the rate-up timer was not recommended in [4]. As shown in [7], over a wide range (from several seconds to about one minute) the rate-up timer value does not have a significant impact on performance and the resulting throughput

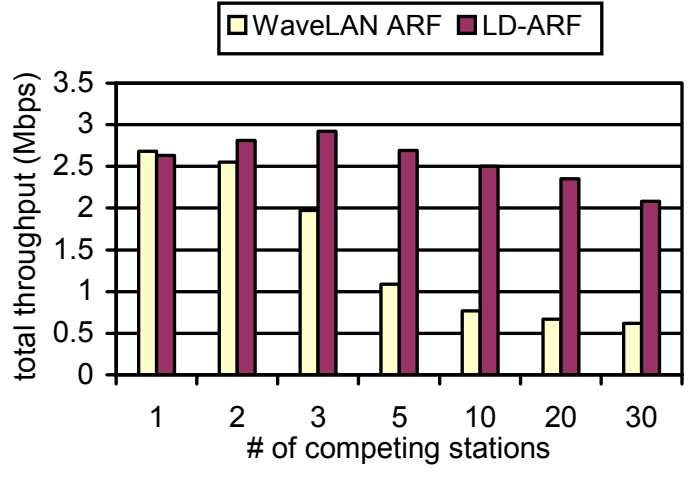

(a) Basic

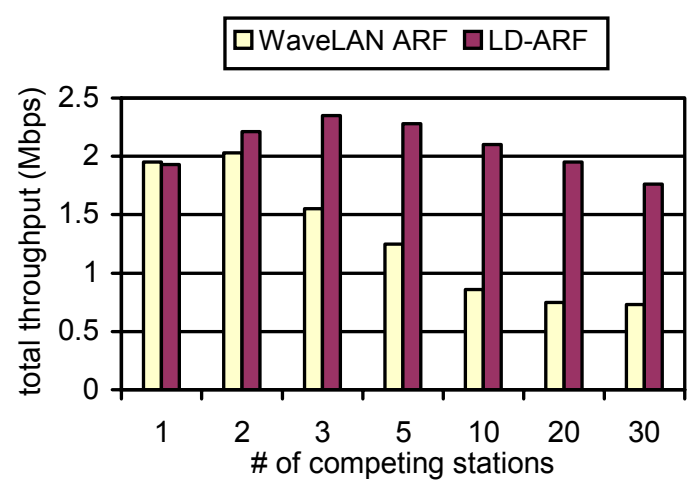

(b) RTS/CTS

Figure 6. Performance comparison of auto rate algorithms - scenario 1

is comparable. In our simulations, we do not put focus on this timer and simply set its value to 10 seconds.

We first consider a situation where all the WLAN links experience exactly the same channel state at the same time (Scenario 1). It can be seen from Figure 6 that when there is only one link (i.e., one station sending traffic), the two algorithms produce similar throughput. However, when there are multiple links (i.e., multiple competing stations), the performance of the original ARF algorithm degrades dramatically. When the number of links is 3 , the performance improvement by the new LD-ARF over ARF is already very significant. When the number of competing stations is large, the new LD-ARF algorithm yields more than double the throughput of the ARF algorithm.

In the following scenarios, we consider more realistic situations where the channel conditions are independent.

In Scenario 2, each link experiences a channel condition that is determined independently from the 10-state channel model. Figure 7 compares the system throughput of LD-ARF and ARF for basic access and RTS/CTS access in Scenario 2. Similar to Scenario 1, when the number of competing stations is large, the throughput improvement by the new LD-ARF algorithm is more than $100 \%$.

In Scenario 3, we consider a case where the stations are separated into two classes. Half of the stations always 


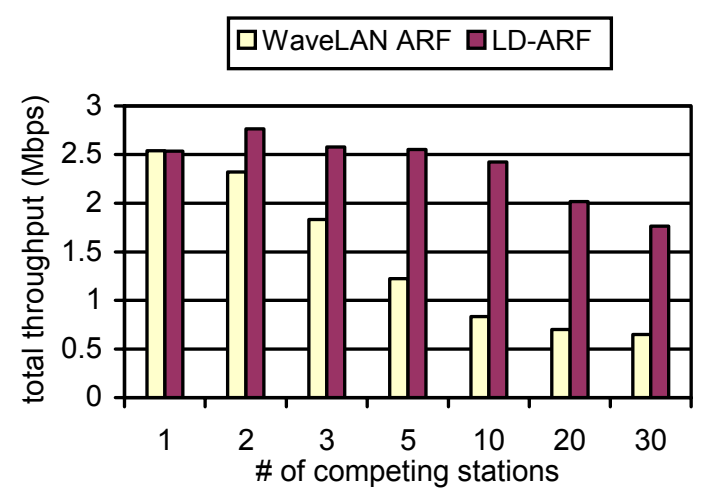

(a) Basic

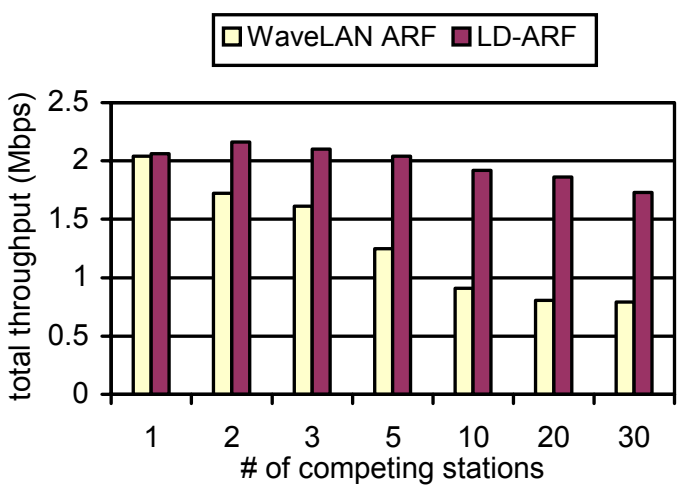

(b) RTS/CTS

Figure 7. Performance comparison of auto rate algorithms - scenario 2

experience the "bad" channel states, i.e., $\mathrm{S}_{0}$ to $\mathrm{S}_{4}$, while the other half always experience the "good" states, i.e., $\mathrm{S}_{5}$ to $\mathrm{S}_{9}$.

The throughput comparison in Figure 8 shows that LDARF still outperforms the original ARF.

Besides throughput, we also compare their proportional fairness indices. The proportional fairness index $(P F I)$ is defined as [24]

$$
P F I=\prod_{i} S_{i}
$$

where $S_{i}$ is the throughput (in Kbps) of the $i$ th station.

The advantage of using PFI over other fairness indices is that PFI can measure not only fairness but also the overall benefit in throughput. Note that fairness does not mean equality. The higher the PFI value, the better the overall performance of a system. Figure 9 compares the PFI between LD-ARF and ARF. When the total number of traffic sending stations is large (e.g., 15 "good" and 15 "bad" stations), LDARF improves PFI by about 11 orders of magnitude over ARF.

The simulation results presented in Figure 6 to Figure 9 reveal that the ARF algorithm cannot perform well in the presence of multiple stations. The reason is mainly due to the misinterpretation of a collision loss to be a link error loss. Therefore in the original ARF, the data rate is inappropriately

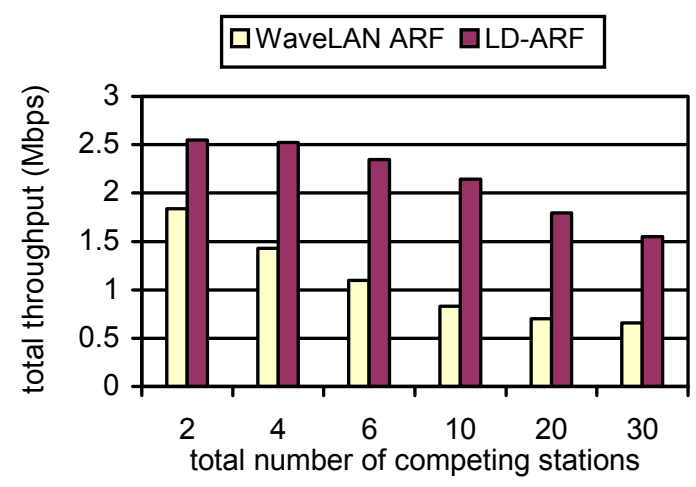

(a) Basic

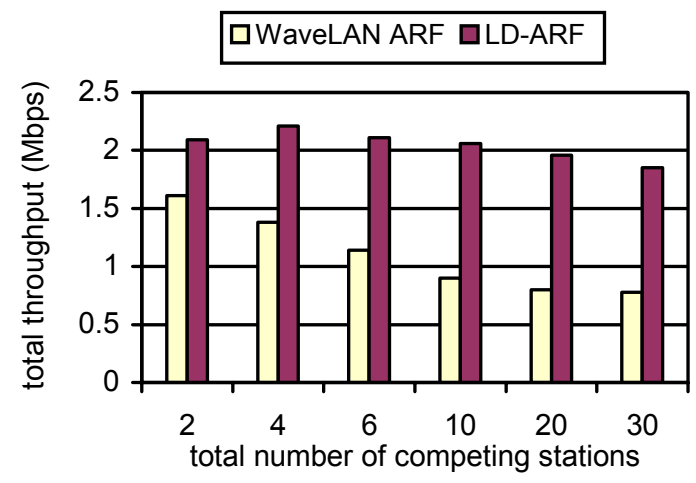

(b) RTS/CTS

Figure 8. Throughput comparison of auto rate algorithms - scenario3

decreased. Let us investigate whether it is possible to eliminate the problem by increasing the threshold value of $N_{\text {down }}$.

Figure 10 gives the comparison of the two auto rate algorithms when $N_{\text {down }}=N_{u p}=10$ (Scenario 4). The channel model for the wireless links is the same as that for Scenario 2, i.e., identical and independent 10-state channel model.

It can be seen from Figure 10 that when there is only one station, the throughput of ARF $N_{\text {down }}=10$ is even lower compared with ARF with $N_{\text {down }}=2$. For other cases, the throughput of ARF is slightly improved compared with the case with $N_{\text {down }}=2$ (Scenario 2). However, compared with LDARF, the performance of ARF is still substantially reduced. When the number of competing stations is increased, the performance of ARF degrades rapidly as in the other scenarios.

The simulation results indicate that the approach of increasing $N_{\text {down }}$ for ARF does not work well. In contrast, LDARF performs well in all the cases we have considered, which demonstrates that it is an efficient and effective approach to overcome the problem of coexistence of two different types of losses in WLANs.

\section{CONCLUSIONS}

In this paper we have investigated the problem experienced by the existing auto rate algorithms in IEEE 802.11 WLANs with multiple stations generating heavy traffic. A new LD-ARF algorithm with loss-differentiation ability is proposed to more 
口WaveLAN ARF QLD-ARF

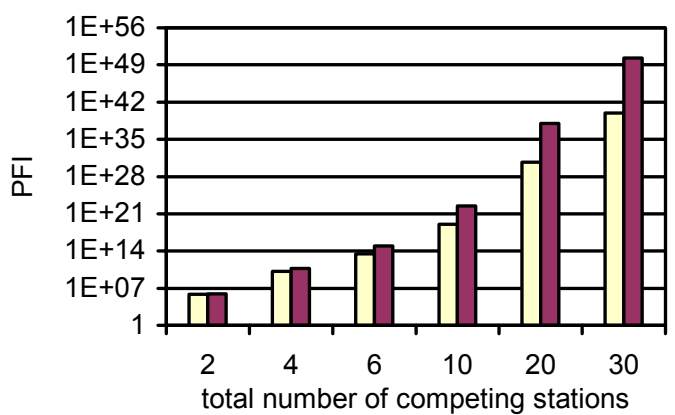

(a) Basic

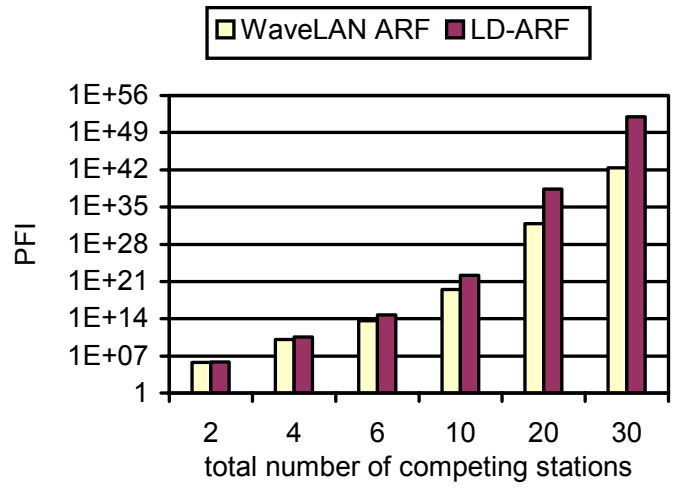

(b) RTS/CTS

Figure 9. Proportional fairness comparison of auto rate algorithms scenario 3

accurately perform the rate adaptation procedure. Comparisons with the original ARF show that more than $100 \%$ improvement in throughput can be achieved by LD-ARF when the number of competing stations is large.

To the best of our knowledge, this paper is the first to address the loss differentiation issue in auto rate algorithms for 802.11 WLAN. Some of the future work is worth mentioning here.

The LD-ARF algorithm proposed in this paper is a straightforward combination of the original ARF algorithm with the loss differentiation MAC. The loss differentiation method and its application to the ARF algorithm involve MAC layer modifications only and this is one of its advantages. However, as we have discussed, the MAC layer loss differentiation is probabilistic. It is possible to further enhance both the loss differentiation method and corresponding auto rate algorithm. If the receiver is powerful enough to extract more physical layer information, e.g., RSS values of the received frames (either successful or erroneous) and embed this information into the control frames, the sender may be able to determine the link quality and contention intensity more accurately. For instance, if RSS is high but the frame is in-error, it is likely that a collision has occurred when the frame is erroneous. If RSS is low, it is more likely that the link quality is bad. By combining both PHY layer information and the modified MAC layer schemes, it is possible to further improve

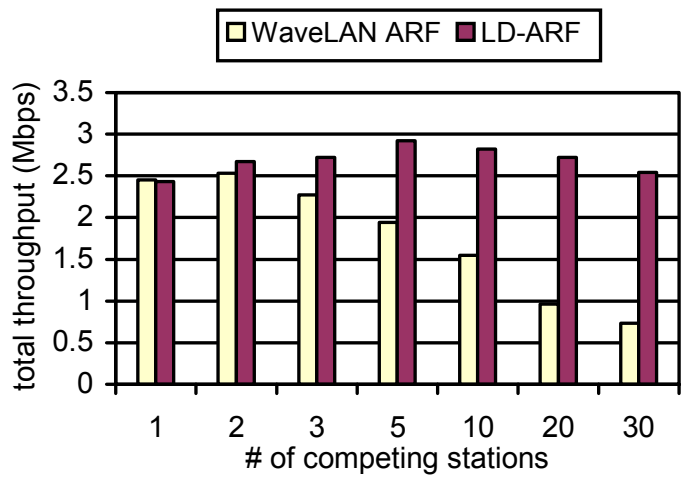

(a) Basic

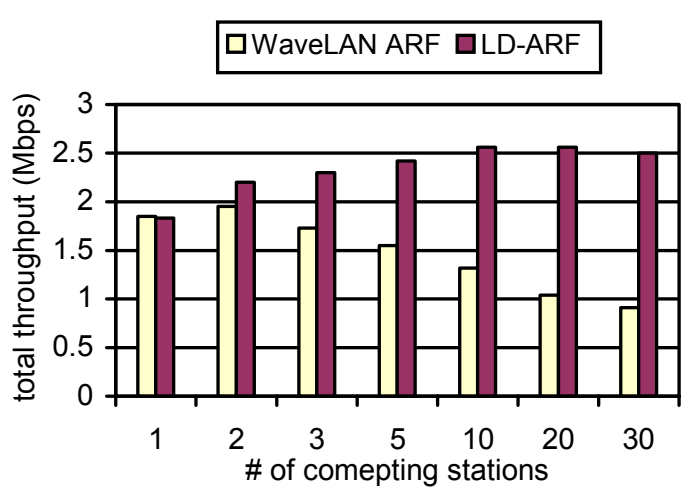

(b) RTS/CTS

Figure 10. Performance comparison of auto rate algorithms - scenario 4

the method proposed in this paper and those RSS or SNR based methods in [6]-[9].

Last but not least, how to adapt the LD-MAC and LD-ARF algorithms discussed in this paper into the on-going 802.11n and 802.11e standards for the future WLAN needs to be further investigated.

\section{ACKNOWLEDGMENT}

This work was sponsored by the Canadian Natural Sciences and Engineering Research Council through grant STPGP 257684-02 and the AoE scheme under the University Grant Committee of Hong Kong (AoE/E-01/99).

\section{APPENDIX: BER FUNCTIOINS OF 802.11B WLANS}

The following BER functions (see Figure 11) are based on [11][12][13] and assume an AWGN channel.

For $802.11 \mathrm{~b}$ at $1 \mathrm{Mbps}$, the relation between $B E R$ and $S N R$ (or SIR) is given by

$$
B E R_{1} \approx Q(\sqrt{11 \times S N R})
$$

For $802.11 \mathrm{~b}$ at $2 \mathrm{Mbps}$, the $B E R$ is given by

$B E R_{2} \approx Q(\sqrt{5.5 \times S N R})$ 
For $802.11 \mathrm{~b}$ at $5.5 \mathrm{Mbps}$, the $B E R$ is given by

$$
B E R_{5.5} \approx \frac{8}{15} \times[14 \times Q(\sqrt{8 \times S N R})+Q(\sqrt{16 \times S N R})]
$$

For $802.11 \mathrm{~b}$ at $11 \mathrm{Mbps}$, the $B E R$ is given by

$$
B E R_{11} \approx \frac{128}{255} \times\left[\begin{array}{l}
24 \times Q(\sqrt{4 \times S N R})+16 \times Q(\sqrt{6 \times S N R})+ \\
174 \times Q(\sqrt{8 \times S N R})+16 \times Q(\sqrt{10 \times S N R})+ \\
24 \times Q(\sqrt{12 \times S N R})+Q(\sqrt{16 \times S N R})
\end{array}\right]
$$

\section{REFERENCES}

[1] M.S. Gast, 802.11 Wireless Networks: The Definitive Guides, O'Reilly, 2002.

[2] IEEE Std 802.11-1999, "Wireless LAN medium access control (MAC) and physical layer (PHY) specifications," Jun. 2003.

[3] IEEE Std 802.11b-1999, "Wireless LAN medium access control (MAC) and physical layer (PHY) specifications: higher-speed physical layer extension in the $2.4 \mathrm{GHz}$ band," Sep. 1999.

[4] A. Kamerman, L. Monteban, "WaveLAN-II: a high-performance wireless LAN for the unlicensed band," Bell Labs Technical Journal, vol.2, no.3, pp.118-133, Aug. 1997.

[5] B.E. Braswell, J.C. McEachen, "Modeling data rate agility in the IEEE 802.11a WLAN protocol," OPNETWORK 2001, March 2001.

[6] G. Holland, N. Vaidya, P. Bahl, "A rate-adaptive MAC protocol for multi-hop wireless networks," ACM MOBICOM 2001, pp.236-250, July 2001.

[7] D. Qiao, S. Choi, K.G. Shin, "Goodput analysis and link adaptation for IEEE 802.11a wireless LANs," IEEE Transactions on Mobile Computing, vol.1 no. 4, pp.278-292, Oct.-Dec. 2002.

[8] J.P. Pavon, S. Choi, "Link adaptation strategy for IEEE 802.11 WLAN via received signal strength measurement," IEEE ICC 2003, pp.11081113, May 2003.

[9] I. Hatatcherev, K. Langendoen, R. Lagendijk, H. Sips, "Hybrid rate control for IEEE 802.11," ACM MobiWac 2004, pp.10-18, Oct. 2004.

[10] M. Lacage, M. H. Manshaei, T. Turletti, "IEEE 802.11 rate adaptation: a practical approach," ACM MSWiM 2004, pp. 126-134, Oct. 2004.

[11] IEEE Std 802.15.2, "Coexistence of wireless personal area networks with other wireless devices operating in unlicensed frequency bands," Aug. 2003.

[12] T.S. Rappaport, Wireless Communications: Principles and Practices, Prentice Hall, 1996.

[13] J.G. Proakis, Digital Communications, McGraw-Hill, 1995.

[14] NS-2, URL http://www.isi.edu/nsnam/ns.
[15] G. Bianchi, "Performance analysis of the IEEE 802.11 distributed coordination function," IEEE Journal on Selected Areas in Communications, vol.18, no.3, pp.535-547, Mar. 2000.

[16] Y. C. Tay, K. C. Chua, "A capacity analysis for the IEEE 802.11 MAC protocol," Wireless Networks, vol.7, no.2, pp.159-171, Mar. 2001.

[17] F. Cali, M. Conti, E. Gregori, "IEEE 802.11 protocol: design and performance evaluation of an adaptive backoff mechanism," IEEE Journal on Selected Areas in Communications, vol.18, no.9, pp.17741786, Sep. 2000.

[18] Q. Pang, S.C. Liew, V. Leung, "Design of an Effective LossDistinguishable MAC Protocol for 802.11 WLAN," to appear in IEEE Communications Letters.

[19] ATM Forum, "ATM user-network interface specification V3.0," Sep. 1993.

[20] E.N. Gilbert, "Capacity of a burst-noise channel," Bell Systems Technical Journal, vol. 39, pp.1253-1265, Sep. 1960.

[21] E.O. Elliot, "Estimates of error rates for codes on burst-noise channels," Bell Systems Technical Journal, vol. 42, pp.1977-1997, Sep. 1963.

[22] A. Willig, M. Kubisch, C. Hoene, A. Wolisz, "Measurements of a wireless link in an industrial environment using an IEEE 802.11compliant physical layer," IEEE Transactions on Industrial Electronics, vol. 43, no.6, pp. 1265-1282, Dec. 2002.

[23] H. S. Wang, N. Moayeri, "Finite-state Markov channel - a uselful model for radio communication channels," IEEE Transactions on Vehicular Technology, vol.44, no.1, pp. 163-171, Feb. 1995.

[24] F. Kelly, "Charging and rate control for elastic traffic," European Transactions on Telecommunications, vol.8, pp.237-252, Jan. 1997.

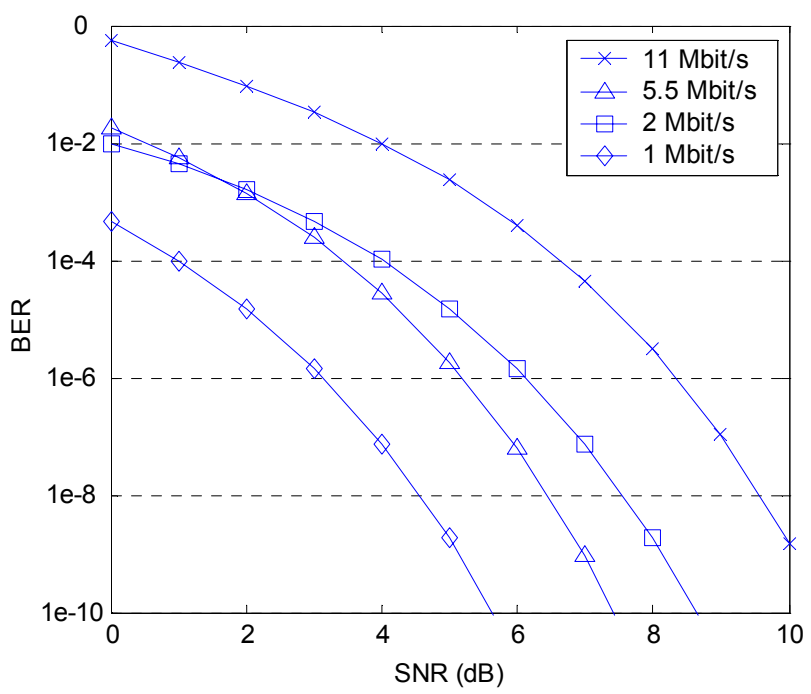

Figure 11. BER versus SNR (or SIR) for $802.11 \mathrm{~b}$ modulation types 